\section{$\underline{\text { Static Mode Microfluidic Cantilevers for Detection of Waterborne Pathogens }}$}

Helen Bridle ${ }^{1,2}$, Wenxing Wang ${ }^{1}$, Despoina Gavriilidou ${ }^{2}$, Farid Amalou ${ }^{1}$, Duncan P. Hand $^{3}$, Wenmiao Shu ${ }^{1}$

1) Institute of Biological Chemistry, Biophysics and Bioengineering, School of Engineering and Physical Sciences, Heriot-Watt University, EH14 4AS

2) School of Engineering, University of Edinburgh, King's Buildings, EH9 3JJ

(when the work was done); now Imperial College London

3) Institute of Photonics and Quantum Sciences, School of Engineering and Physical Sciences, Heriot-Watt University, EH14 4AS

Corresponding author: h.l.bridle@hw.ac.uk; 01314513355

ABSTRACT: This paper reports on the first demonstration of polymeric microfluidic cantilever sensors. Microcantilever sensors, magnetic beads, and microfluidic technology has been combined to create a polymer based biosensor. Using cheap materials like polyimide, a simple fabrication method has been developed to produce cantilevers with an embedded microfluidic channel. The advantage of this approach is that the addition of a microfluidic channel enables the analysis of smaller volumes and increases the capture efficiency in applications detecting rare analytes. As a proof of principle the system has been applied for the detection of the waterborne protozoan parasite Cryptosporidium, achieving sensitivity comparable to QCM, whereas a previous set-up without the microfluidic channel was unable to detect the parasite.

Keywords: microfluidics, cantilever, Cryptosporidium, detection 
Cantilever biosensors have demonstrated impressive sensitivity for the detection of nuclei acids, proteins and cells [1-4]. However, in solution, when

41 operated in the resonance mode, viscous damping severely degrades the resolution [5]. Alternatively, cantilevers can be operated in static mode, with surface stress determining the degree of cantilever bending. While this eliminates the problem of viscous damping for measurements in liquid, the challenge then becomes effective delivery of the sample to the cantilever surface. This challenge is especially important in applications where relatively large analyte sample volumes are necessary, e.g. environmental monitoring [6]. In order to address this, immobilisation strategies can be optimised to attempt to maximise capture efficiency of the sensor or external forces can be utilised to enhance delivery [7].

Previously, cantilevers have been embedded within microfluidic systems [8, 9]; and more recently, smaller-scale microfluidics which fits onto the cantilever surface itself is demonstrated. For example, the Manalis group have developed microfluidics upon cantilevers, manufactured from silicon and employed in the resonance mode. This highly successful strategy has lead to the weighing of single cells in fluid [5]. Very few other microfluidic cantilever systems have been reported [10]. However, the materials and fabrication approaches are expensive. Additionally, while the latter work provides an interesting method of weighing individual microorganisms, specificity in pathogen detection is not offered.

Cryptosporidium is a protozoan pathogen, which is highly problematic for the water industry due to a low infectious dose [11] and high degree of robustness which enables long survival times in water along with resistance to standard disinfection by chlorination [12]. Several biosensor technologies have been applied to the detection of Cryptosporidium as reported in a recent review article [13]. Both quartz crystal microbalance (QCM) [14] and piezoelectric macrocantilever (PEMC) [15] approaches utilised relatively large flow cells and delivery of the sample to the sensor surface was not characterised. 
Here we present the low-cost manufacture of polymeric microfluidic cantilevers and demonstrate the effectiveness of this set-up in improving transport to the sensor in both the detection of pathogens and DNA. The approach reported here has the advantage of ensuring effective sample delivery to the surface of the sensor, enabling high capture efficiency, which is useful in the situation of detecting rare pathogens. Miniaturisation of sample delivery in this way limits the throughput of devices, although there is potential to negate this problem through parallelisation or effective sample pre-processing. Previous unpublished work by the authors using microcantilevers without microfluidic channels presented low sensitivity to Cryptosporidium oocysts whereas use of the microfluidic channel has enabled a detection limit of $1 \times 10^{5}$ oocysts/mL. However, the main advantage of the system presented here over previous microfluidic cantilever set-ups is that since the device is made entirely of polyimide it is both cheaper and easier to manufacture.

83

\section{Materials and Methods}

\subsection{Cantilever manufacture}

The sensor was precision fabricated using a photolithography method. Firstly, a sheet of polyimide (7.6 micron thick, 3 inch x 50 inch,VHGLABS Kapton ${ }^{\circledR}$ (Polymide)) was sputter-coated with an adhesive layer of chrome $(5 \mathrm{~nm})$ followed by a layer of gold $(20 \mathrm{~nm})$ using gold evaporation system (BOC Edwards Auto 500). Secondly, this gold-coated polyimide was attached to a sheet of $20 \mu \mathrm{m}$ thick positive photoresist (photopolymer dry film resist, ORDYL), and the two sheets were bonded together using pressure applied at $95^{\circ} \mathrm{C}$. Thirdly, a mask (fabricated by microlithography) was employed to control the UV exposure (exposure time of 30 seconds) creating patterns of microchannels. Fourthly, the UV exposed sheet was developed (Developer conc. for 4615 dry film Mega Electronics Ltd) for 20 seconds removing the positive photoresist in the exposed areas. These areas define the microfluidic channels. Fifthly, the microchannels were sealed using $25 \mu \mathrm{m}$ polyimide tape as a top layer. This process is summarised in Figure 1A. Finally, a short pulsed (65 ns) laser of wavelength $532 \mathrm{~nm}$ was used to cut the structures into individual microcantilever microfluidic chips, with cantilever dimensions of $1.5 \mathrm{~mm}$ in length and $300 \mu \mathrm{m}$ in width. Each cantilever contained one $\mathrm{U}$ shaped microfluidic channel 
104 with channel sizes of $60 \mu \mathrm{m}$ in width, $20 \mu \mathrm{m}$ in height and total of $3 \mathrm{~mm}$ in length

105 (Figure 1B).

106

2.2 Cantilever Set-Up and Operation

108

109 The cantilever set-up developed in this paper includes a rotary valve, microcantilever

110 chip with a microchannel fabricated on top that is connected with tubing to a gravity

111 fed pumping system (1) via the rotary valve (2), laser diode (7), position-sensitive

112 detector (PSD) (8), a magnet and microscope with a digital CCD camera (9) (Figure

$1131 \mathrm{C}$; numbers relate to the labels in Figure 1C). The cantilever system is set up on an

114 optical table (4) (Newport Laminar Flow isolator) to reduce vibrations. The system is

115 mounted in a non-transparent box (3) made of PMMA (5mm thickness), with thermal

116 insulated materials (10mm thickness), which reduces the external disturbance from air

117 flow, background light, and temperature variations in the lab \pm 00.5 degree]. The

118 rotary valve switch device is computer-controlled via RS-232 and is used to switch

119 between the flow different liquids into the microchannel on the cantilever surface.

120 With the use of the rotary valve, in addition to gravity pumping of the liquid $(1 \mathrm{~mL} / \mathrm{h})$,

121 spikes in the results curve can be significantly reduced. The optical resolution of the

122 microscope is $5 \mu \mathrm{m}$, which is used to confirm that the laser beam is on the tip of the

123 cantilever. The laser beam reflected by the cantilever is aligned on to a position-

124 sensitive detector (PSD) and an amplifier is used to amplify the current signal from

125 the PSD and convert into voltage signals. A National Instrument data acquisition card

126 is then used to record data in LabView.

127

\subsection{Detection of Cryptosporidium}

129

130 Reagents: Viable C. parvum oocysts were purchased from Creative Science

131 Company, Moredun Research Institute. Magnetic beads and goat polyclonal antibody

132 immunoglobulin $\mathrm{G}$ (IgG) specific to C. parvum were purchased from Waterborne Inc.

133 Phosphate-buffered saline (PBS) was obtained from Sigma-Aldrich.

135 Functionalization of cantilever microfluidic biosensor with protein G, antibody IgG

136 and immobilization with $C$. parvum solution: The sensor was functionalized with 
protein $\mathrm{G}$ solution $(20 \mathrm{mg} / \mathrm{mL})$ for 2 hours, $\operatorname{IgG}$ solution $(20 \mu \mathrm{g} / \mathrm{mL})$ for another 2

138 hours [16] and finally exposed to C. parvum solution (between $1 \times 10^{5}$ oocysts $/ \mathrm{mL}$ and

$1391 \times 10^{7}$ oocysts $/ \mathrm{mL}$ in DI water) for 10 mins causing the oocysts' immobilization on the

140 surface of the sensor. After each step was complete, the sensor was rinsed with PBS

141 solution $(10 \mathrm{mM}, \mathrm{pH} 7,4)$. After immobilization of oocysts, the biosensor was left to

142 stabilize and afterwards it was incubated with magnetic beads solution (Crypto-Grab,

143 Waterborne Inc, $2.5 \mathrm{mg} / \mathrm{mL}$ ) for 20 minutes. Finally the sensor was rinsed with PBS

144 solution. Every rinsing was performed in order to remove the unbound reagents. The

145 protocol was performed in room temperature. The flow rate for all steps was $1 \mathrm{~mL} / \mathrm{hr}$.

\section{Results and Discussion}

\subsection{Cantilever manufacture}

152 Microfluidic channels embedded in silicon cantilevers have previously been 153 manufactured using dry etching. In order to utilise low-cost polyimide materials an 154 alternative fabrication method was required for the production of microfluidic 155 channels. A method using simple lithographic techniques was employed, as described 156 in detail in the materials and methods, and illustrated in Figure 1B.

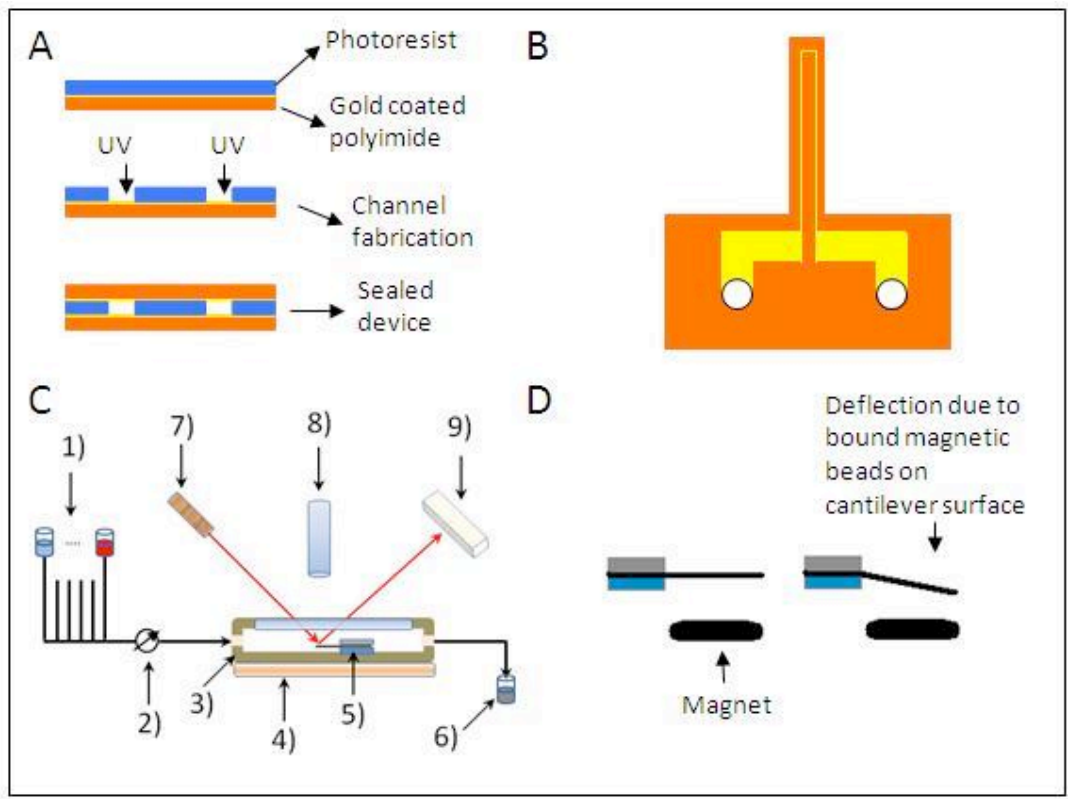

159 Figure 1.Scheme of integrated microfluidic microcantilever sensor. A) Schematic of the fabrication 


\subsection{Cantilever Characterisation}

Following production of the cantilevers, the system was characterised using optical microscopy. Figure 2A shows an optical microscope image of polyimide fabricated cantilevers with embedded microchannels. The width of the cantilever was designed to be $300 \mu \mathrm{m}$ and the channel is $60 \mu \mathrm{m}$ wide. Images from several cantilevers were taken, and an average of $X$ measurements revealed the channel width was $X \mu \mathrm{m} \pm 3 \mu \mathrm{m}$, illustrating that the variability in fabrication was small and that this is therefore a reproducible method. The images illustrate that cantilevers of different lengths can be manufactured using this protocol, though for all subsequent experiments cantilevers of length $1.5 \mathrm{~mm}$ were employed.

In the cantilever set-up illustrated in Figure 1C cantilever performance was tested. Flow through the microfluidic channel had no influence upon deflection with the cantilever remaining stable. Various flow rates were trialled and an upper of limit of $1 \mathrm{~mL} / \mathrm{hr}$ was determined. This was limited primarily by the choice to operate using gravity driven flow. While the bonding technique could tolerate higher pressures, and therefore flow rates, pumping of fluids through the channel was observed to result in spikes in the cantilever read-out. 


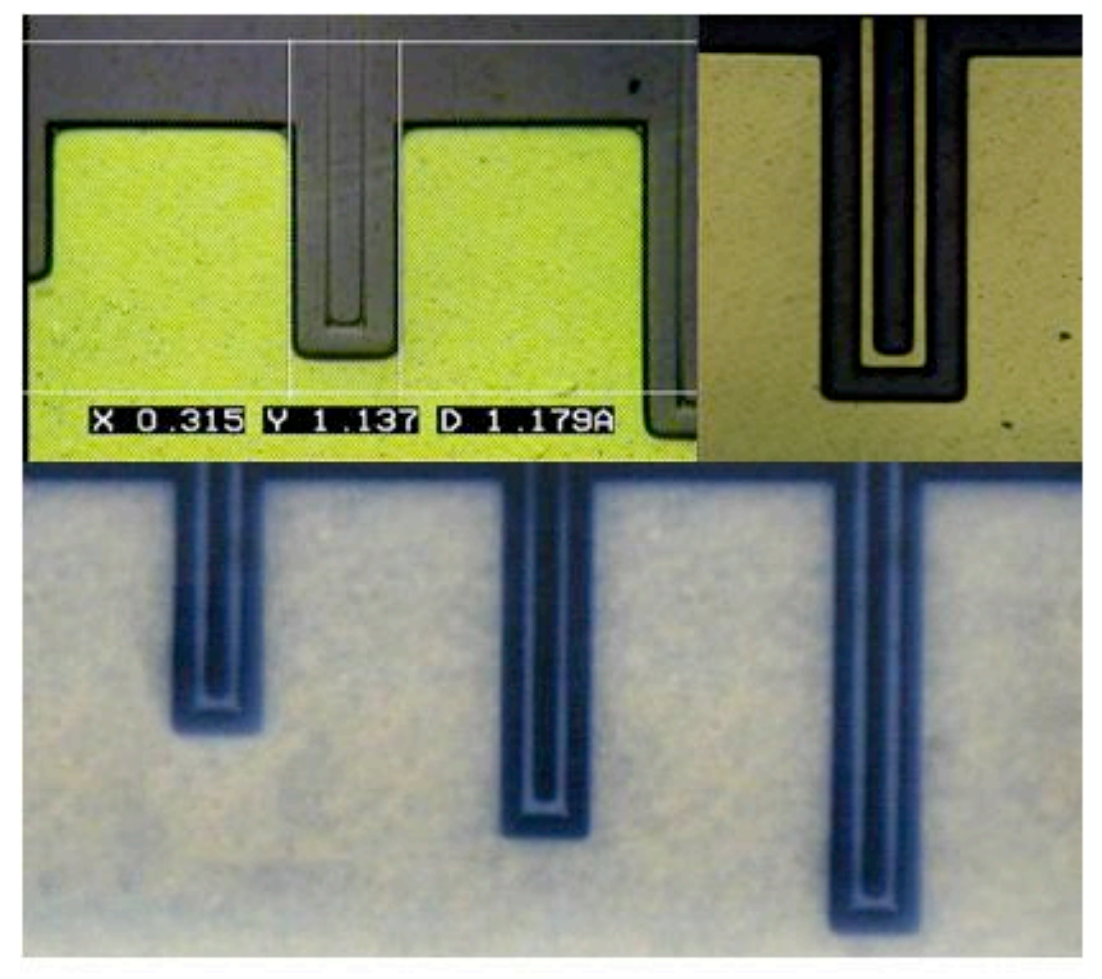

Figure 2. Cantilever characterisation. Optical microscope images of fabricated microchannels on microcantilevers.

The final performance characterisation involved system calibration with magnetic beads (Figures 1D and 2B). Figure 3A illustrates the schematic of detection employed for the waterborne parasite under investigation. Detection of whole cells is

191 challenging in mass-sensitive systems as coupling of the binding event to the system 192 deflection is critical and this is often weak for larger analytes like cells. Additionally, 193 factors such as surface stress also contribute to the observed signal. Therefore, the use 194 of magnetic beads was selected to amplify the signal. Figure 1D illustrates the 195 operation and set-up with this detection principle with a magnet located beneath the 196 cantilever holder. To determine that the magnet strength and magnetic bead 197 concentration were appropriate a series of experiments flowing different 198 concentrations of magnetic beads through the system were performed. As seen in 199 Figure 2B, quantitative results were obtained with a series of dilutions indicating that 200 the cantilever read-out was proportional to the magnetic bead concentration within the 201 channel, thus confirming this approach was suitable for quantitative pathogen 202 detection. 


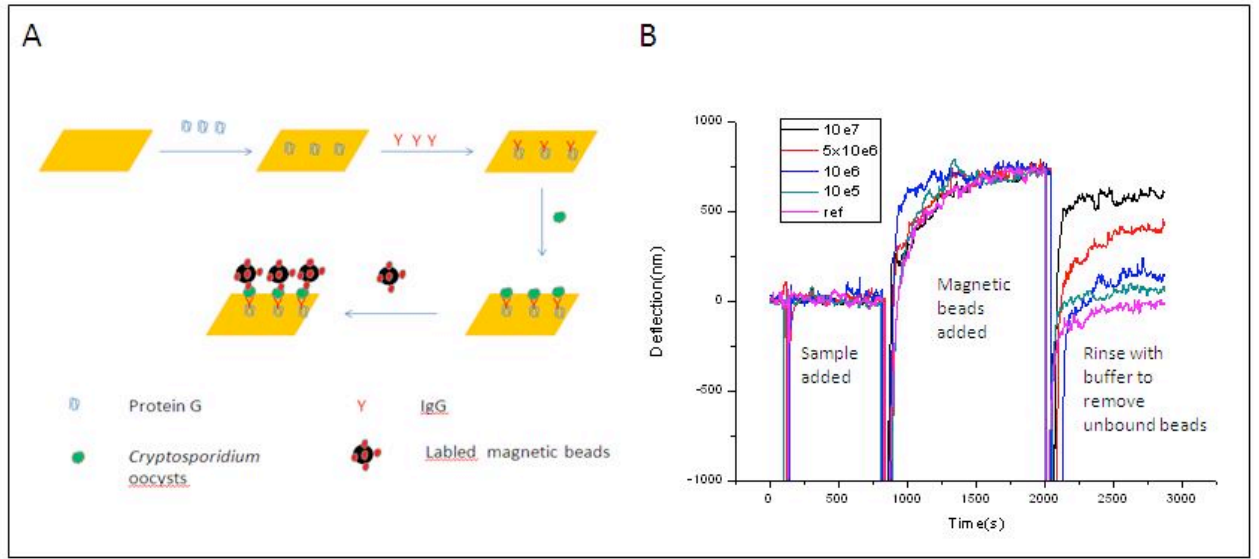

204

Figure 3. Cantilever detection of waterborne pathogens. A) Schematic illustrating the functionalisation of the cantilever to detect Cryptopsoridium oocysts and the addition of magnetic beads which enables enhancement of the detection signal. B) Detection of oocysts at a range of different concentrations ranging from a control sample of zero to a set of concentrations from $1 \times 10^{5}$ to $1 \times 10^{7}$ oocysts. Initially the oocysts solution is passed through the cantilever microchannel and although binding takes place this is insufficient to trigger cantilever bending. After the introduction of the sample a brief rinsing step with PBS is applied. Subsequently, magnetic beads are passed through the channel (at this stage where the beads are incubated in the channel little difference is observed between different oocyst concentrations) and finally the channel is rinsed with buffer removing any unbound beads. In the final stage of the results curve, the measurement of deflection indicates the amount of bound microbeads, and therefore also the concentration of oocysts within the cantilever channel, and it is clear that the biosensor can distinguish between different concentrations of pathogen.

\subsection{Pathogen Detection}

The microfluidic cantilever system was applied to the detection of the waterborne protozoan pathogen, Cryptosporidium. Detection of this pathogen is challenging since it is often present at low concentrations. However, since ingestion of only a few oocysts is sufficient to cause disease it is important to maximise capture efficiency of oocysts within any biosensor system.

Our initial work (unpublished) exploring the potential of cantilever sensors to detect this pathogen were unpromising with the parasite going undetected even at high concentrations. The most likely explanation for this was the sample size and time required for delivery of the pathogen to the surface. Since, an identical set-up was employed during cantilever functionalisation, limitations in delivery of one of the 
immobilisation reagents and/or the antibody to the surface might also have

231 contributed to the poor detection.

The time allowed for oocyst exposure to the surface was 10 mins. In the set-up 234 without a flow system using $1 \mathrm{~mL}$ of solution the time was insufficient to result in a 235 high capture efficiency on the cantilever surface. The time taken, $t$, for a particle to 236 diffuse a distance, $\mathrm{d}$, is given by:

$$
d \sim \sqrt{D t}
$$

Equation 1

where $\mathrm{D}$ is the diffusion coefficient $\left(5 \times 10^{-10} \mathrm{~cm}^{2} / \mathrm{s}\right.$ for oocysts) [6], [17]. This would suggest that oocysts diffuse around $0.002 \mathrm{~mm}$ in ten minutes.

However, consideration of diffusion may not be appropriate for oocysts as it has been reported that for micron-sized particles [24], hydrodynamic and gravitational forces are often significant compared to Brownian forces [18]. In the static case, hydrodynamic forces are not relevant and the gravitational force can be determined using the particle free settling velocity, $\mathrm{U}_{\mathrm{s}}$. This is given by:

$$
U_{s}=\frac{2 \Delta \rho g \alpha^{2}}{9 \mu}
$$

\section{Equation 2}

247 where $\Delta \rho\left(\mathrm{kg} / \mathrm{m}^{3}\right)$ is the particle density (1045.4) minus the density of water (997), $\mathrm{g}$ 248 is the acceleration of gravity $\left(9.81 \mathrm{~m} / \mathrm{s}^{2}\right), \alpha$ is the particle radius $(2.5 \mu \mathrm{m}$ for $\mathrm{C}$. 249 parvum) and $\mu$ is the water viscosity $\left(8.91 \times 10^{-4} \mathrm{~kg} / \mathrm{ms}\right)$, and is $0.74 \mu \mathrm{m} / \mathrm{s}$ for $C$. 250 parvum. Our calculated figure compares to the slightly lower values of 0.35 and 0.5 $251 \mu \mathrm{m} / \mathrm{s}$ reported in the literature. Although oocyst travel by sedimentation is around an 252 order of magnitude greater than that of diffusion, and additionally is focused in the 253 direction of the substrate, this is still unlikely to enable efficient delivery of oocysts to 254 the cantilever surface within ten minutes, since using an average of the above values 255 of $0.53 \mu \mathrm{m} / \mathrm{s}$, allows for a distance of only $0.31 \mathrm{~mm}$ to be covered. If a test volume of $2560.1 \mathrm{~mL}$ was utilised it would take days (assuming the volume was solely located on 257 top of the cantilever). However, the non-flow set-up also has the disadvantage that in 258 the flow cell set-up, which is wider, longer and deeper than the cantilever, many 259 oocysts will initially be distributed under or to the sides of the cantilever and therefore 260 be unable to reach the binding surface, especially allowing for sedimentation. Oocysts 261 could not be detected even after $1 \mathrm{hr}$. 
Within the microfluidic cantilever set-up, both diffusion and settling are still

264 valid methods of oocyst transport to the surface within the channel laminar flow 265 environment. Given the volume of the channel $(0.0036 \mu \mathrm{L})$ and the flow rate $(1$ $266 \mathrm{~mL} / \mathrm{hr}$ ) it is clear that the transit time within the channel is much less than $1 \mathrm{~s}$. With a 267 channel height of $20 \mu \mathrm{m}$ the maximal distance (in the $\mathrm{z}$ direction) to be travelled by 268 an oocyst within this time is $10 \mu \mathrm{m}$ (allowing for the size of the oocyst). It must 269 however be remembered that there is an even distribution of oocysts across the 270 channel height and many will need to travel significantly less than this distance to 271 reach the binding surface. While it is clear that not all oocysts will reach the surface 272 even in the microfluidic cantilever set-up the chances are greatly improved. Increasing 273 the number of encounters with the immobilised antibodies increases the likelihood of 274 a binding event occurring and will therefore increase the capture efficiency of the 275 system.

With the microfluidic cantilever system a series of different Cryptosporidium 278 concentrations $\left(10^{5}\right.$ to $10^{7}$ oocysts $\left./ \mathrm{mL}\right)$ were investigated, with each concentration 279 repeated five times. Following capture of the oocysts, the system was flushed with 280 magnetic beads to amplify the signal. Figure 3B shows representative traces of the 281 experiments, from the oocyst addition stage until the final detection point at which the 282 unbound magnetic beads are removed from the system. One trace for each 283 concentration is shown along with a reference sample where no Cryptosporidium was 284 added. As the magnetic beads flow through the system little difference is observed 285 between the different samples. However, upon rinsing of the magnetic beads from the 286 system the reference sample returns to zero, whereas for the oocyst samples magnetic 287 beads remain bound to oocysts within the system and can be utilised to determine the 288 Cryptosporidium concentration in the sample. In short, Figure 3B illustrates that 289 quantitative detection of oocysts can occur within the range $10^{5}$ to $10^{7}$ oocysts $/ \mathrm{mL}$.

The results of all five experiments have been averaged and are presented in 292 Figure 4 . The results indicate a linear relationship $\left(\mathrm{R}^{2}=0.96\right)$ confirming detection in 293 the range $10^{5}$ to $10^{7}$ oocysts $/ \mathrm{mL}$. The upper limit of $10^{7}$ oocysts $/ \mathrm{mL}$ was the highest 294 concentration tested in this set-up and could potentially be extended. This is limited 295 by the space for oocyst binding within the microchannel. Interestingly, a calculation 
of the maximum coverage of the microchannel area revealed that it would be saturated with $\sim 1 \times 10^{6}$ oocysts, using an oocyst diameter of $5 \mu \mathrm{m}$, a channel area of 18 $\mathrm{mm}^{2}$ (assuming oocysts only bind to the immobilised antibody and not to other channel surfaces) and assuming a maximum close-packing of $74 \%$. This calculation reveals that although the use of the microchannel improves the capture efficiency, the system still misses some oocysts. By decreasing the flow rate more time would be available for oocysts to bind within the channel, thus increasing the sensitivity. There is thus a trade-off between reaching highly sensitive detection limits and achieving a reasonable throughput/detection time, which is a recurring challenge for biosensor system for waterborne pathogens.
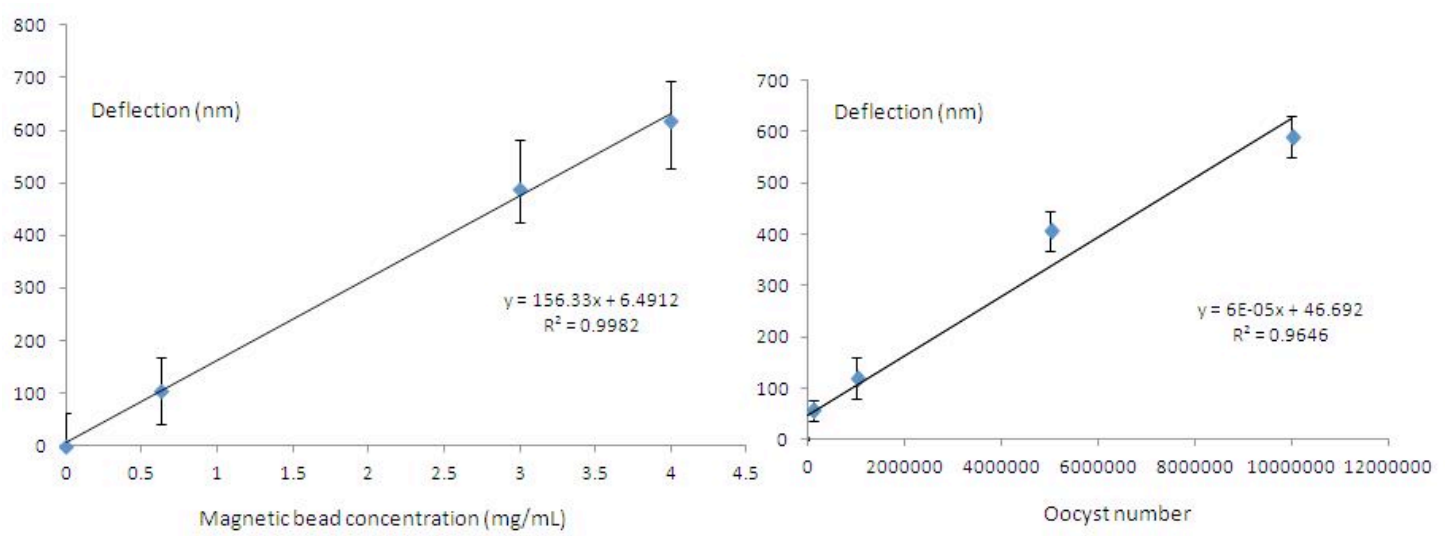

Figure 4. Plot of deflection (nm) against magnetic bead concentration (left graph) and oocyst number (confirming that the magnetic enhancement of detection is quantitative) and oocyst exposure.

For practical applications, achieving a low limit of detection is the critical

313 parameter. Lower concentrations were found not to yield a measurable response.

314 While the sensitivity of the approach is comparable to the $1 \times 10^{5}$ oocysts $/ \mathrm{mL}$ detection 315 limit reported for QCM-D detection of this parasite (Poitras 2009), lower 316 concentrations have been determined, by Mutharasan and colleagues (Campbell 317 2008), with a macrocantilever set-up. However, this operates with a recirculating flow 318 system, which could potentially also increase the capture efficiency of the 319 microcantilever sensor. Additionally, sensitivity could be improved by increasing the magnetic bead concentration or utilising a more powerful magnet. 


\section{Conclusions}

The results in this paper represent the first example of a microfluidic 324 microcantilever sensor fabricated in polyimide. Using polymer materials to 325 manufacture the system is an advance over previous work, allowing for cheap and 326 easy fabrication, resulting in cheap sensors which can be rapidly produced. A further 327 advantage of this approach relates to the effective sample delivery enabled by 328 confining the sample to a narrow layer above the cantilever surface. Transport of the 329 analyte of interest to the capture region is often the time-limiting step and this design 330 offers a mechanism of effective surface delivery. This is likely to prove advantageous 331 for applications detecting rare analytes as well as in applications where very small 332 samples are to be processed. For larger samples throughput within the microfluidic 333 channels is potential challenge though parallelisation is an option to overcome this 334 possible limitation. Future work could incorporate cantilever sensors on the ends of 335 optical fibres moving towards a miniaturised portable system [19].

Furthermore, this paper has applied the system for the detection of the problematic waterborne protozoan parasite Cryptosporidium, demonstrating sensitivities comparable to existing literature reports and particularly showing greater sensitivity than QCM. Future work will concentrate on the optimisation of the system as well as

341 developments in the immobilisation chemistry and the sample pre-processing to 342 deliver even lower limit of detection, suitable for real-world application of this 343 technology to waterborne pathogen detection.

Acknowledgements: HB would like to acknowledge the Royal Academy of

346 Engineering/EPSRC for her Fellowship.

\section{References}

1. Liu, D. and W. Shu, Microcantilever Biosensors: Probing Biomolecular 485. 
2. Goeders, K.M., J.S. Colton, and L.A. Bottomley, Microcantilevers: Sensing Chemical Interactions via Mechanical Motion. Chemical Reviews, 2008. 108: p. $522-542$.

3. Takahashi, H., et al., Differential pressure sensor using a piezoresistive cantilever. Journal of Micromechanics and Microengineering, 2012. 22: p. 055015.

4. Rasmussen, P.A., A.V. Grigorov, and A. Boisen, Double sided surface stress cantilever sensor. Journal of Micromechanics and Microengineering, 2005. 15: p. 1088.

5. Burg, T.P., et al., Weighing of biomolecules, single cells and single nanoparticles in fluid. Nature, 2007. 446(7139): p. 1066-1069.

6. Squires, T.M., R.J. Messinger, and S.R. Manalis, Making it stick: convection, reaction and diffusion in surface-based biosensors. Nat Biotechnol, 2008. 26(4): p. 417-426.

7. Liju, Y., Dielectrophoresis assisted immuno-capture and detection of foodborne pathogenic bacteria in biochips. Talanta, 2009. 80(2): p. 551-558.

8. Johansson, A., et al., SU-8 cantilever sensor system with integrated readout. Sensors and Actuators A: Physical, 2005. 123: p. 111-115.

9. Calleja, M., et al., Polymeric mechanical sensors with piezoresistive readout integrated in a microfluidic system in Microtechnologies for the New Millennium 2003. 2003: 7th International Conference on Miniaturized Chemical and Biochemlcal Analysts Systems

10. Ricciardi, C., et al., Integration of microfluidic and cantilever technology for biosensing application in liquid environment. Biosensors and Bioelectronics, 2010. 26(4): p. 1565-1570.

11. Okhuysen, P.C., et al., Virulence of three distinct Cryptopsoridium parvum isolates for healthy adults. Infectious Diseases, 1999. 180: p. 1275-1281.

12. King, B.J. and P.T. Monis, Critical processes affecting Cryptosporidium oocyst survival in the environment. Parasitology, 2007. 134: p. 309-323.

13. Bridle, H., et al., Detection of Cryptosporidium in miniaturised fluidic devices. Water Research, 2012. 46(6): p. 1641-1661.

14. Poitras, C., J. Fatisson, and N. Tufenkji, Real-time microgravimetric quantification of Cryptosporidium parvum in the presence of potential interferents. Water Research, 2009. 43(10): p. 2631-2638.

15. Campbell, G.A. and R. Mutharasan, Near real-time detection of Cryptosporidium parvum oocyst by IgM-functionalized piezoelectric-excited millimeter-sized cantilever biosensor. Biosensors and Bioelectronics, 2008. 23(7): p. 1039-1045.

16. Gavriilidou, D. and H. Bridle, Comparison of immobilisation strategies for Cryptosporidium parvum immunosensors. Biochemical Engineering Journal, 2012. 68: p. 231-235.

17. Gervaise, P. and P. Molin, The role of water in solid-state fermentation. Biochemical Engineering Journal, 2003. 13: p. 85-101.

18. Yiantsios, S.G. and A.J. Karabelas, Deposition of micron-sized particles on flat surfaces: effects of hydrodynamic and physicochemical conditions on particle attachment efficiency. Chemical Engineering Science, 2003. 58(14): p. 3105-3113.

19. Albri, F., et al., Laser machining of sensing components on the end of optical fibres. Journal of Micromechanics and Microengineering, 2012. 23(4): p. 045021. 
404 